\title{
Expression of synovial fluid biomarkers in patients with knee osteoarthritis and meniscus injury
}

\author{
JIAN DING $^{1}, \mathrm{XIN} \mathrm{NIU}^{2}$, YAN SU$^{1}$ and XIAOLIN LI ${ }^{1}$ \\ ${ }^{1}$ Department of Orthopaedic Surgery and ${ }^{2}$ Institute of Orthopaedic Surgery, Shanghai Jiao Tong University \\ Affiliated Sixth People's Hospital, Shanghai 200233, P.R. China
}

Received February 3, 2017; Accepted May 12, 2017

DOI: 10.3892/etm.2017.4636

\begin{abstract}
In the present study, the levels of synovial fluid biomarkers of patients with knee osteoarthritis (OA) and those with meniscus injury (MI) were compared to associate the levels of synovial fluid biomarkers with the degree of OA and MI. Synovial fluid samples were obtained from 51 cases with OA and 40 patients with MI. Severity of OA and MI were evaluated using the Kellgren-Lawrence (K-L) classification and Magnetic Resonance Imaging Osteoarthritis Knee Score, respectively. A comparative analysis of the levels of matrix metalloproteinase-13 (MMP-13), vascular endothelial growth factor (VEGF), interleukin (IL)-10, IL-8, IL-6, IL-1, tumor necrosis factor- $\alpha$ (TNF- $\alpha$ ), as well as collagenase 2 in synovial fluid was made between patients with OA and MI. We found that synovial fluid levels of VEGF and IL-6 were significantly higher in patients with OA than in patients with MI, and IL-10 was lower in patients with OA compared to MI patients $(\mathrm{p}<0.05)$. After adjusting for sex, course of disease, and surgical history, no significant associations between K-L scores and biomarker levels were found for patients with OA. In the MI patients, TNF- $\alpha$ was significantly associated with magnetic resonance imaging (MRI) score. In conclusion, patients with knee OA and MI have different patterns of biomarker expression in their synovial fluid.
\end{abstract}

\section{Introduction}

The two most common knee joint disorders involving cartilage damage are knee osteoarthritis (OA) and meniscus injury (MI). $\mathrm{OA}$, which is an age-related disease with increasing incidence due to increase in the aging population (1), is characterized by loss and damage of articular cartilage and other structures surrounding the joint: ligaments, synovial membrane, subchondral bone and menisci (2). OA pathogenesis is poorly known,

Correspondence to: Dr Jian Ding, Department of Orthopaedic Surgery, Shanghai Jiao Tong University Affiliated Sixth People's Hospital, 600 Yishan Road, Shanghai 200233, P.R. China E-mail: dingjian3246@163.com; dh296h@163.com

Key words: collagenase 2, growth factor, inflammatory factors, meniscus injury, osteoarthritis whereas some factors that contribute to the development and progression of OA have been identified encompassing inflammation and altered biomechanical conditions (3).

The menisci are fibro-cartilage-like tissue mainly consisting of collagen and water, with cells interposed (4). They are important for stability, shock absorption, and transmission of load and play a pivotal role in the the normal function and long-term health of the knee joint. As the menisci become worn with age they are more prone to lesions and tears caused by degenerative disease, trauma, or a conjunction of both, leading to significant musculoskeletal morbidity (4). Similar to $\mathrm{OA}, \mathrm{MI}$ is a degenerative disease, the incidence of which correlates with age. MI is considered vital to the development of knee OA and its abnormal condition bears greater risk for the subsequent radiographic OA development (5). The interaction between chondrocytes and the extracellular matrix (ECM) exerts an important role in the pathophysiology of OA (6). Abnormal chondrocyte/ECM interaction leads to the imbalance of destructive cytokines, particularly matrix metalloproteinase-13 (MMP-13), over regulatory factors, resulting in enzymatic degradation of cartilage matrix without adequate inhibition. The balance between matrix synthesis and metalloproteinase inhibition is also crucial for meniscus repair (7). The level of growth factors and MMPs in the synovial fluid may reflect the severity and progression of $\mathrm{OA}$ and MI as the synovial fluid has physical contact directly with structures of the joint and functions to reduce friction during movement (8). Collagenase 2 is involved in the metabolism of subchondral bone (collagen type I) and cartilage (collagen type II). It is a candidate biomarker in the pathological tissue remodeling associated with OA and MI $(9,10)$. Vascular endothelial growth factor (VEGF) serves as a specific angiogenic factor secreted from endothelial cells, synoviocytes and chondrocytes (11). There is a positive association between synovial fluid VEGF and severity of OA. In this study, we compared the levels of inflammatory cytokines, MMPs, collagenase, and VEGF of the synovial fluid in cases with OA and MI and to examine the association between the levels of these synovial proteins and the severity of OA and MI.

\section{Patients and methods}

The study was a case-control study (level III evidence). During the prospective cross-sectional study, patients with OA and 


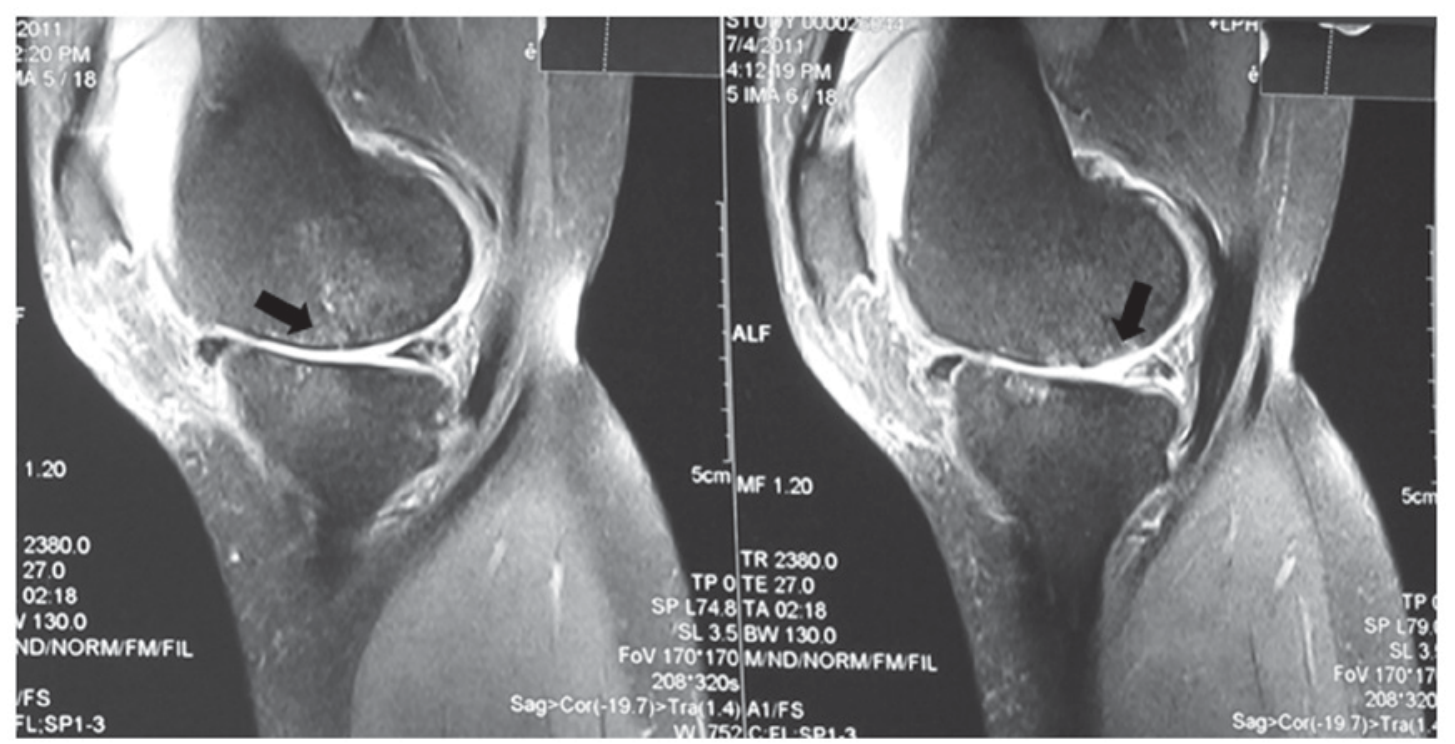

Figure 1. MRI image of OA (male, 72 years). The arrows shows the articular cartilage and meniscus wear in the knee joint. MRI, magnetic resonance imaging; OA, osteoarthritis.

MI provided informed consent and were then recruited by the Shanghai Jiao Tong University Affiliated Sixth People's Hospital. The present study obtained the approval from the Ethics Committee of Shanghai Jiao Tong University Affiliated Sixth People's Hospital.

The inclusion criteria were as follows: patients with symptomatic primary knee OA diagnosed based on the American College of Rheumatology criteria or patients with MI diagnosed based on magnetic resonance imaging (MRI) findings of an abnormally shaped meniscus and a strong signal intensity at the meniscus on MRI images (12). Patients with OA and MI were excluded. All the patients received consensus diagnosis from three senior orthopedic surgeons. Radiological grading (Kellgren-Lawrence score, K-L score) (13) and Stoller grade, assessed by MRI, were also confirmed by at least two of the three senior orthopedic surgeons. The exclusion criteria were as follows: patients with OA or MI who had received immunosuppressive drugs, patients who had received intra-articular sodium hyaluronate injection treatment, patients who had previous operations for OA or trauma, or patients with other combined inflammatory or neurodegenerative disease. Patients with a Stoller grade I MI were also excluded.

From January 2010 to December 2014, 91 patients were enrolled for the present study, 51 with OA and 40 with MI. All the patients underwent anterior-posterior and lateral X-ray, and MRI of the knee. The classic MRI images of OA and MI are shown in Figs. 1 and 2. Medical and surgical histories were surveyed. Synovial fluid was collected from each patient via arthrocentesis immediately before the first injection in the current course of sodium hyaluronate treatment or before a scheduled operation.

Synovial fluid studies. Arthrocentesis of the affected knee was performed on all the patients to collect $2 \mathrm{ml}$ synovial fluid samples prior to the first therapeutic sodium hyaluronate injection of the current treatment course or prior to operation. Centrifugation at at $1,200 \mathrm{xg}$ for $5 \mathrm{~min}$ was implemented on the samples and then they were stored at a temperature

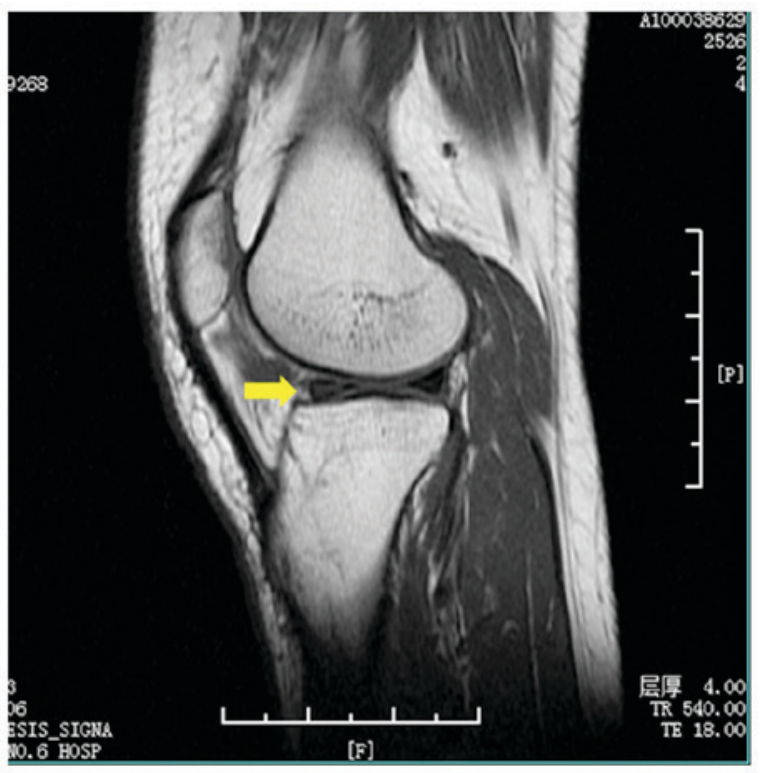

Figure 2. MRI image of MI (male, 32 years). The arrow shows the meniscus injury in the knee joint. MRI, magnetic resonance imaging; MI, meniscus injury.

of $-80^{\circ} \mathrm{C}$. Synovial fluid samples were assayed for VEGF, tumor necrosis factor- $\alpha$ (TNF- $\alpha$ ), MMP-13, interleukin (IL)-1, IL-6, IL-8, IL-10 and collagenase 2 using commercial ELISA kits (R\&D Systems, Inc., Minneapolis, MN, USA) according to the manufacturer's instructions.

Statistical analysis. Demographic characteristics of OA and MI patients were analyzed. Range and median were obtained for continuous variables, and the percentage and frequency were shown for categorical variables. Assessment of OA was carried out using X-ray K-L classification score (14), while assessment of MI was carried out with the use of a semi-quantitative scoring method based on MRI (15). The association between K-L score and biochemical markers, including VEGF, TNF- $\alpha$, MMP-13, 
Table I. Characteristics of patients with OA and MI.

\begin{tabular}{lc}
\hline A, Patients with OA $(\mathrm{N}=51)$ & \\
\hline Characteristics & Values \\
\hline Age (years), median (range) & $63(44-79)$ \\
Male, N (\%) & $14(27)$ \\
Disease duration (months), median (range) & $12(8-240)$ \\
Biochemical markers, median (range) & \\
VEGF (pg/ml) & $0.71(0.30-1.18)$ \\
TNF- $\alpha$ (pg/ml) & $0.44(0.15-0.83)$ \\
MMP-13 (ng/ml) & $0.19(0.15-0.43)$ \\
IL-10 (pg/ml) & $0.13(0.08-0.72)$ \\
IL-8 (pg/ml) & $0.27(0.13-1.89)$ \\
IL-6 (pg/ml) & $0.75(0.23-3.04)$ \\
IL-1 (pg/ml) & $0.09(0.06-0.34)$ \\
Collagenase 2 (ng/ml) & $3.14(1.90-3.49)$ \\
Previous operation, N (\%) & \\
Yes & $0(0)$ \\
No & $51(100)$ \\
K-L score, N (\%) & \\
I & $18(35)$ \\
II & $17(33)$ \\
III & $16(32)$ \\
\hline
\end{tabular}

B, Patients with MI $(\mathrm{N}=40)$

\begin{tabular}{lc} 
Age (years), median (range) & $32(16-40)$ \\
Male, N $(\%)$ & $18(45)$ \\
Disease duration (months), median (range) & $9(1-19)$ \\
Biochemical markers, median (range) & \\
VEGF (pg/ml) & $0.51(0.16-1.23)$ \\
TNF- $\alpha(\mathrm{pg} / \mathrm{ml})$ & $0.42(0.15-0.73)$ \\
MMP-13 (ng/ml) & $0.19(0.16-0.40)$ \\
IL-10 (pg/ml) & $0.17(0.08-0.97)$ \\
IL-8 (pg/ml) & $0.21(0.10-1.43)$ \\
IL-6 (pg/ml) & $0.48(0.13-3.00)$ \\
IL-1 (pg/ml) & $0.09(0.06-0.66)$ \\
Collagenase 2 (ng/ml) & $3.13(1.39-3.53)$ \\
Previous operation, N (\%) & \\
Yes & $5(13)$ \\
No & $35(87)$ \\
MRI level, N $(\%)$ & \\
I & $2(5)$ \\
II & $14(35)$ \\
III & $24(60)$ \\
\hline
\end{tabular}

OA, osteoarthritis; MI, meniscus injury; VEGF, vascular endothelial growth factor; TNF, tumor necrosis factor; MMP, matrix metalloproteinase; IL, interleukin; K-L score, Kellgren-Lawrence score; MRI, magnetic resonance imaging.

IL-10, IL-8, IL-6, IL-1 and collagenase 2, were evaluated by ordinal logistic (cumulative logistic) regression analysis (16),
Table II. Estimates from the ordinal logistic regression of biochemical marker values (log-transformed) to K-L score for OA patients, with higher B-value indicating tendency towards higher K-L score.

\begin{tabular}{lccc}
\hline Biochemical markers & B-value & SE & P-value \\
\hline VEGF $(\mathrm{pg} / \mathrm{ml})$ & 0.13 & 0.97 & 0.89 \\
TNF- $\alpha(\mathrm{pg} / \mathrm{ml})$ & -0.81 & 0.73 & 0.27 \\
MMP-13 (ng/ml) & -0.25 & 1.33 & 0.85 \\
IL-10 (pg/ml) & -0.65 & 0.81 & 0.42 \\
IL-8 (pg/ml) & 0.83 & 0.86 & 0.34 \\
IL-6 (pg/ml) & -0.32 & 0.52 & 0.54 \\
IL-1 (pg/ml) & 0.89 & 1.13 & 0.43 \\
Collagenase 2 (ng/ml) & -0.31 & 3.25 & 0.92 \\
\hline
\end{tabular}

Analysis for each biochemical marker is adjusted for sex, disease duration and operation history. K-L score, Kellgren-Lawrence score; OA, osteoarthritis; SE, standard error; VEGF, vascular endothelial growth factor; TNF, tumor necrosis factor; MMP, matrix metalloproteinase; IL, interleukin.

adjusting for sex, disease duration and operation history. Similarly, the association between MRI score and biochemical markers were assessed. Linear regression analysis was then used to examine the mean difference in values of biochemical markers between the OA and MI patients, adjusting for sex, disease duration and operation history. Since biochemical marker values were heavily skewed, they were log-transformed when we performed association/difference analysis. Analyses were performed using the statistical software SPSS version 20 (IBM SPSS, Armonk, NY, USA).

\section{Results}

Table IA and B shows the characteristics of OA and MI patients. The gender and age distributions between the two patient groups were notably different, the OA group was older and included more women.

As shown in Table II, after adjusting for sex, disease duration, and operation history, no biochemical markers were found to be significantly associated with the K-L score in OA patients. The biochemical marker TNF- $\alpha$ was significantly correlated with MRI score in MI patients (Table III). Table IV shows the mean difference in log-transformed biochemical marker values between OA and MI patients, adjusting for sex, disease duration and operation history. We found that the mean log-transformed IL-6 value significantly differed between $\mathrm{OA}$ and MI patients $(\mathrm{P}<0.05)$. Specifically, OA patents had significantly greater IL- 6 value. In addition, the OA patents had marginally significantly smaller IL-10 values.

\section{Discussion}

The present study revealed that patients with OA had significantly higher VEGF and IL-6 levels, and lower IL-10 levels in their synovial fluid as compared to those with MI. VEGF is a potent proangiogenic factor. An in vivo study has shown that 
Table III. Statistical results of the ordinal logistic regression between synovial fluid levels of biochemical markers and K-L scores for patients with OA.

\begin{tabular}{lrcc}
\hline Biochemical markers & B-value & SE & P-value \\
\hline VEGF $(\mathrm{pg} / \mathrm{ml})$ & -2.65 & 1.49 & 0.07 \\
TNF- $\alpha(\mathrm{pg} / \mathrm{ml})$ & 2.92 & 1.36 & $0.03^{\mathrm{a}}$ \\
MMP-13 (ng/ml) & 0.78 & 2.17 & 0.72 \\
IL-10 (pg/ml) & 0.54 & 1.15 & 0.64 \\
IL-8 (pg/ml) & 0.84 & 1.10 & 0.44 \\
IL-6 (pg/ml) & 0.77 & 1.04 & 0.46 \\
IL-1 (pg/ml) & 0.74 & 2.01 & 0.71 \\
Collagenase 2 (ng/ml) & -12.20 & 7.08 & 0.09 \\
\hline
\end{tabular}

SE, standard error; VEGF, vascular endothelial growth factor; TNF, tumor necrosis factor; MMP, matrix metalloproteinase;

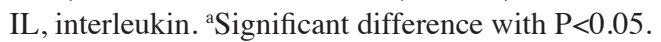

primary human chondrocytes incubated in synovial fluids of patients with OA actively secrete VEGF (14), suggesting a role for inflammation and hypoxia in OA pathogenesis. In addition, intra-articular VEGF injection in healthy mice led to symptoms including synovial hyperplasia, calcification in articular cartilage, as well as bone sclerosis, followed by cartilage degradation, indicative of OA (17). However, in animal models of meniscal lesions, the local application of VEGF did not increase angiogenesis in avascular or vascular regions of meniscus or improve meniscal healing (18). Consistent with these findings, a high level of VEGF was observed in synovial fluids from patients with OA which was not observed in that from patients with MI.

IL-6 is a protein involved in cartilage degradation in vitro and stimulates nociceptors in patients with OA (19). Recent findings have demonstrated that patients with end-stage knee OA have significantly higher levels of IL-6 in synovial fluids as compared with control donors (20), supporting the hypothesis that inflammatory processes are involved in OA. Similarly, another study has shown that the levels of IL-6 in the synovial fluid from patients with OA and those with symptomatic cartilage defects are identical, and this level is significantly higher than those of healthy volunteers (21). More IL-6 is produced in cartilage regeneration by osteoarthritic chondrocytes compared to healthy and defective chondrocytes. Furthermore, adding IL-6 to healthy chondrocytes in vitro increased glycosaminoglycan production, but adding IL-6 to osteoarthritic chondrocytes led to decreased production (22). These results suggest IL-6 as a key player in cartilage matrix production, and that suppressing IL-6 in synovial fluids may improve cartilage repair in patients who have OA or symptomatic cartilage defects. Our finding that the levels of IL-6 in synovial fluid from patients with OA are higher than those in patients with MI adds to our current knowledge regarding the levels of IL-6 in the synovial fluid from patients with MI.

IL-10, serving as a pleiotropic immunoregulatory cytokine, has chondroprotective effects, and the promoting effect on chondrocyte proliferation and on hypertrophic or
Table IV. Statistical results of the ordinal logistic regression between synovial fluid levels of biochemical markers and MOAKS for patients with meniscus injury.

\begin{tabular}{lccc}
\hline Biochemical markers & $\begin{array}{c}\text { Mean } \\
\text { difference }\end{array}$ & SE & P-value \\
\hline VEGF (pg/ml) & 0.33 & 0.09 & 0.24 \\
TNF- $\alpha(\mathrm{pg} / \mathrm{ml})$ & 0.01 & 0.09 & 0.13 \\
MMP-13 (ng/ml) & 0.00 & 0.05 & 0.43 \\
IL-10 (pg/ml) & -0.15 & 0.10 & 0.10 \\
IL-8 (pg/ml) & 0.13 & 0.11 & 0.19 \\
IL-6 (pg/ml) & 0.55 & 0.15 & $0.02^{\text {a }}$ \\
IL-1 (pg/ml) & -0.06 & 0.07 & 0.38 \\
Collagenase 2 (ng/ml) & 0.03 & 0.03 & 0.97 \\
\hline
\end{tabular}

SE, standard error; VEGF, vascular endothelial growth factor; TNF, tumor necrosis factor; MMP, matrix metalloproteinase; IL, inter-

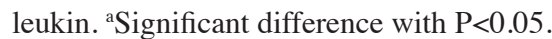

chondrogenic differentiation by means of activating the bone morphogenetic protein (BMP) signaling pathway. In OA, the level of IL-10 in cartilage and the synovium are elevated (23). Studies on synovial fluid levels of IL-10 in patients with MI are limited, and our study shows that patients with MI have higher levels of IL-10 in their synovial fluids in relation to patients with OA. In our study, MI patients were younger than OA patients. It is possible higher IL-10 reflects better control of joint inflammation in younger populations. However, the underlying mechanism of how IL-10 relates to OA and MI remains to be elucidated.

In our study, any significant correlation between the $\mathrm{K}-\mathrm{L}$ score of patients with $\mathrm{OA}$ or any of the investigated biomarkers (VEGF, TNF- $\alpha$, MMP-13, IL-10, IL-8, IL-6, IL-1 and collagenase 2) was not found. However, a significant correlation was observed between the MRI Osteoarthritis Knee Score (MOAKS) value and TNF- $\alpha$ level in MI patients. These results suggest that levels of these biomarkers cannot reliably differentiate the severity of OA and MI, which explains the lack of reported associations.

One possible limitation of our study is single sampling of biomarkers in synovial fluid. The wide range in the levels of biomarkers suggests that future studies should rely on multiple sampling to avoid possible false results. Our study was also limited by patient number, which may not have been sufficient to detect true differences in levels of cytokine or growth factor in synovial fluid between OA and MI.

In conclusion, this study compared a series of synovial fluid biomarkers from patients with OA and MI. Patients with OA had higher VEGF and IL-6 levels, and lower levels of IL-10 than that found in patients with MI, indicating different biomarker patterns in these pathologies.

\section{Acknowledgements}

The authors would like to thank Dr Jie Mao for editing the manuscript. The study was supported by Chinese National Nature Science Foundation (grant 81171861). 


\section{References}

1. Benazzo F, Perticarini L, Padolino A, Castelli A, Gifuni P, Lovato M, Manzini C and Giordan N: A multi-centre, open label, long-term follow-up study to evaluate the benefits of a new viscoelastic hydrogel (Hymovis ${ }^{\circledR}$ ) in the treatment of knee osteoarthritis. Eur Rev Med Pharmacol Sci 20: 959-968, 2016.

2. Bijlsma JW, Berenbaum F and Lafeber FP: Osteoarthritis: an update with relevance for clinical practice. Lancet 377 : 2115-2126, 2011

3. Maldonado M and Nam J: The role of changes in extracellular matrix of cartilage in the presence of inflammation on the pathology of osteoarthritis. Biomed Res Int 2013: 284873, 2013.

4. Fox AJ, Wanivenhaus F, Burge AJ, Warren RF and Rodeo SA The human meniscus: a review of anatomy, function, injury, and advances in treatment. Clin Anat 28: 269-287, 2015.

5. Badlani JT, Borrero C, Golla S, Harner CD and Irrgang JJ: The effects of meniscus injury on the development of knee osteoarthritis: data from the osteoarthritis initiative. Am J Sports Med 41: 1238-1244, 2013.

6. Iannone $\mathrm{F}$ and Lapadula $\mathrm{G}$ : The pathophysiology of osteoarthritis. Aging Clin Exp Res 15: 364-372, 2003.

7. Buma P, Ramrattan NN, van Tienen TG and Veth RPH: Tissue engineering of the meniscus. Biomaterials 25: 1523-1532, 2004.

8. Balakrishnan L, Nirujogi RS, Ahmad S, Bhattacharjee M, Manda SS, Renuse S, Kelkar DS, Subbannayya Y, Raju R, Goel R, et al: Proteomic analysis of human osteoarthritis synovial fluid. Clin Proteomics 11: 6, 2014.

9. Karsdal MA, Nielsen MJ, Sand JM, Henriksen K, Genovese F, Bay-Jensen AC, Smith V, Adamkewicz JI, Christiansen C and Leeming DJ: Extracellular matrix remodeling: the common denominator in connective tissue diseases. Possibilities for evaluation and current understanding of the matrix as more than a passive architecture, but a key player in tissue failure. Assay Drug Dev Technol 11: 70-92, 2013.

10. Karsdal MA, Woodworth T, Henriksen K, Maksymowych WP, Genant H, Vergnaud P, Christiansen C, Schubert T, Qvist P, Schett G, et al: Biochemical markers of ongoing joint damage in rheumatoid arthritis - current and future applications, limitations and opportunities. Arthritis Res Ther 13: 215, 2011.

11. Mabey T, Honsawek S, Saetan N, Poovorawan Y, Tanavalee A and Yuktanandana P: Angiogenic cytokine expression profiles in plasma and synovial fluid of primary knee osteoarthritis. Int Orthop 38: 1885-1892, 2014

12. Rubin DA and Paletta GA Jr: Current concepts and controversies in meniscal imaging. Magn Reson Imaging Clin N Am 8: 243-270, 2000.
13. Tanishi N, Yamagiwa H, Hayami T, Mera H, Koga Y, Omori G and Endo N: Relationship between radiological knee osteoarthritis and biochemical markers of cartilage and bone degradation (urine CTX-II and NTX-I): the Matsudai Knee Osteoarthritis Survey. J Bone Miner Metab 27: 605-612, 2009.

14. Hunter DJ, Guermazi A, Lo GH, Grainger AJ, Conaghan PG, Boudreau RM and Roemer FW: Evolution of semi-quantitative whole joint assessment of knee OA: MOAKS (MRI Osteoarthritis Knee Score). Osteoarthritis Cartilage 19: 990-1002, 2011.

15. Hoff P, Buttgereit F, Burmester GR, Jakstadt M, Gaber T, Andreas K, Matziolis G, Perka C and Röhner E: Osteoarthritis synovial fluid activates pro-inflammatory cytokines in primary human chondrocytes. Int Orthop 37: 145-151, 2013

16. McCullagh P and Nelder JA: Generalized Linear Models. Vol 37. 2nd edition. Chapman \& Hall, New York, NY, 1989.

17. Ludin A, Sela JJ, Schroeder A, Samuni Y, Nitzan DW and Amir G: Injection of vascular endothelial growth factor into knee joints induces osteoarthritis in mice. Osteoarthritis Cartilage 21: 491-497, 2013.

18. Kopf S, Birkenfeld F, Becker R, Petersen W, Stärke C, Wruck CJ, Tohidnezhad M, Varoga D and Pufe T: Local treatment of meniscal lesions with vascular endothelial growth factor. J Bone Joint Surg Am 92: 2682-2691, 2010.

19. Lee AS, Ellman MB, Yan D, Kroin JS, Cole BJ, van Wijnen AJ and Im HJ: A current review of molecular mechanisms regarding osteoarthritis and pain. Gene 527: 440-447, 2013.

20. Beekhuizen M, Gierman LM, van Spil WE, Van Osch GJ, Huizinga TWJ, Saris DBF, Creemers LB and Zuurmond AM: An explorative study comparing levels of soluble mediators in control and osteoarthritic synovial fluid. Osteoarthritis Cartilage 21: 918-922, 2013

21. Tsuchida AI, Beekhuizen M, Rutgers M, van Osch GJ, Bekkers JE, Bot AG, Geurts B, Dhert WJ, Saris DB and Creemers LB: Interleukin-6 is elevated in synovial fluid of patients with focal cartilage defects and stimulates cartilage matrix production in an in vitro regeneration model. Arthritis Res Ther 14: R262, 2012.

22. Wang M, Shen J, Jin H, Im HJ, Sandy J and Chen D: Recent progress in understanding molecular mechanisms of cartilage degeneration during osteoarthritis. Ann N Y Acad Sci 1240: 61-69, 2011

23. Jung YK, Kim GW, Park HR, Lee EJ, Choi JY, Beier F and Han SW: Role of interleukin-10 in endochondral bone formation: anabolic effect via BMP/Smad pathway. Arthritis Rheum 65: 3153-3164, 2013. 